1 Hacettepe Journal of Mathematics and Statistics

$\bigcap$ Volume $44(4)$ (2015), $839-853$

\title{
Generalization of Hermite-Hadamard type inequalities for $n$-times differentiable functions which are $s$-preinvex in the second sense with applications
}

\author{
Muhammad Amer Latif* ${ }^{* \dagger}$ and Svere S. Dragomir ${ }^{\ddagger \S}$
}

\begin{abstract}
In this paper, Hermite-Hadamard inequality for differentiable preinvex functions is generalized and refined for $n$-times differentiable functions which are $s$-preinvex in the second sense. Some recent results are also improved and applications to special means of positive numbers are given.
\end{abstract}

2000 AMS Classification: 26D07, 26D10, 26D99.

Keywords: Hermite-Hadamard's inequality, invex set, preinvex function, Hölder's inequality, $s$-preinvex function.

Received 16/04/2013 : Accepted 18/04/2014 Doi : 10.15672 /HJMS.2015449438

\section{Introduction}

The following definition for convex functions is well known in mathematical literature:

1.1. Definition. A function $f: I \rightarrow \mathbb{R}, \emptyset \neq I \subseteq \mathbb{R}$, is said to be convex on $I$ if inequality

$$
f(t x+(1-t) y) \leq t f(x)+(1-t) f(y),
$$

holds for all $x, y \in I$ and $t \in[0,1]$.

${ }^{*}$ School of Computational and Applied Mathematics, University of the Witwatersrand, Private Bag 3, Wits 2050, Johannesburg, South Africa, Email: m_amer_latif@hotmail.com

${ }^{\dagger}$ Corresponding Author.

${ }^{\ddagger}$ School of Engineering and Science, Victoria University, PO Box 14428 Melbourne City, MC 8001, Australia,

${ }^{\S}$ School of Computational and Applied Mathematics, University of the Witwatersrand, Private Bag 3, Wits 2050, Johannesburg, South Africa, Email: sever.dragomir@vu.edu.au 
A number of inequalities have been established for convex functions but the following double inequalites, known as Hermite-Hadmard inequalities, are famous in mathematical literature

$$
f\left(\frac{a+b}{2}\right) \leq \frac{1}{b-a} \int_{a}^{b} f(x) d x \leq \frac{f(a)+f(b)}{2},
$$

where $f: I \subseteq \mathbb{R} \rightarrow \mathbb{R}$ is a convex mapping and $a, b \in I \subseteq \mathbb{R}$ with $a<b$. The inequalities (1.1) hold in reversed direction if $f$ is concave. A number of papers have been written on this inequality providing new proofs, noteworthy extensions, generalizations, refinements, counterparts and new Hermite-Hadamard-type inequalities and numerous applications, see for instance [7]-[11], [13], [15], [20]-[23], [29], [31], [32]-[34], [36], [37] and the references therein.

In recent years, the classical convexity has been generalized and extended in a diverse manner. One of them is the preinvexity, introduced by Weir et al. [38] as a significant generalization of convex functions. Many researchers have studied the basic properties of the preinvex functions and their role in optimization, variational inequalities and equilibrium problems, for example see the work of Mohn et al. [24], Noor [26] and Yang et al. [41]. It is well known that the preinvex functions and invex sets may not be convex functions and convex sets respectively.

Let us recall some definitions and known results concerning invexity and preinvexity

1.2. Definition. [41] A set $K \subseteq \mathbb{R}^{n}$ is said to be invex with respect to $\eta: K \times K \rightarrow \mathbb{R}^{n}$ if

$$
x+t \eta(y, x) \in K, \forall x, y \in K, t \in[0,1]
$$

The invex set $K$ is also called an $\eta$-connected set.

1.3. Definition. [38] Let $K \subseteq \mathbb{R}^{n}$ be an invex set with respect to $\eta: K \times K \rightarrow \mathbb{R}^{n}$. A function $f: K \rightarrow \mathbb{R}$ is said to be preinvex with respect to $\eta$, if for all $u, v \in K$ and $t \in[0,1]$, the following inequality holds

$$
f(u+t \eta(v, u)) \leq(1-t) f(u)+t f(v) .
$$

The function $f$ is said to be preincave if and only if $-f$ is preinvex.

It is to be noted that every preinvex function is convex with respect to the map $\eta(x, y)=x-y$ but the converse is not true see for instance [38].

Noor [28], proved the following Hermite-Hadamard type inequalities.

1.4. Theorem. [28] Let $f: K=[a, a+\eta(b, a)] \rightarrow(0, \infty)$ be a preinvex function on the interval of the real numbers $K^{\circ}$ (the interior of $K$ ) and $a, b \in K^{\circ}$ with $\eta(b, a)>0$. Then the following inequalities holds:

$$
f\left(\frac{2 a+\eta(b, a)}{2}\right) \leq \frac{1}{\eta(b, a)} \int_{a}^{a+\eta(b, a)} f(x) d x \leq \frac{f(a)+f(b)}{2} .
$$

Barani et al. in [5], presented the following estimates of the right-side of a HermiteHadamard type inequality in which preinvex functions are involved.

1.5. Theorem. [5] Let $K \subseteq \mathbb{R}$ be an open invex subset with respect to $\eta: K \times K \rightarrow \mathbb{R}$. Suppose that $f: K \rightarrow \mathbb{R}$ is a differentiable function. Assume $p \in \mathbb{R}$ with $p>1$. If $\left|f^{\prime}\right|^{\frac{p}{p-1}}$ 
is preinvex on $K$, for every $a, b \in K$ with $\eta(b, a) \neq 0$, then the following inequality holds:

$$
\begin{gathered}
\left|\frac{f(a)+f(a+\eta(b, a))}{2}-\frac{1}{\eta(b, a)} \int_{a}^{a+\eta(b, a)} f(x) d x\right| \\
\leq \frac{|\eta(b, a)|}{2(1+p)^{\frac{1}{p}}}\left[\frac{\left|f^{\prime}(a)\right|^{\frac{p}{p-1}}+\left|f^{\prime}(b)\right|^{\frac{p}{p-1}}}{2}\right]^{\frac{p-1}{p}} .
\end{gathered}
$$

1.6. Theorem. [5] Let $K \subseteq \mathbb{R}$ be an open invex subset with respect to $\eta: K \times K \rightarrow \mathbb{R}$. Suppose that $f: K \rightarrow \mathbb{R}$ is a differentiable function. If $\left|f^{\prime}\right|$ is preinvex on $K$, for every $a, b \in K$ with $\eta(b, a) \neq 0$, then the following inequality holds:

$$
\begin{aligned}
\mid \frac{f(a)+f(a+\eta(b, a))}{2}-\frac{1}{\eta(b, a)} \int_{a}^{a+\eta(b, a)} & f(x) d x \mid \\
& \leq \frac{|\eta(b, a)|}{8}\left(\left|f^{\prime}(a)\right|+\left|f^{\prime}(b)\right|\right) .
\end{aligned}
$$

Most recently, Li [42] introduced the notion of $s$-preinvexity and established HermiteHadamard type inequalities for this class of functions.

1.7. Definition. [42] Let $K \subseteq[0, \infty)$ be an invex set with respect to $\eta: K \times K \rightarrow \mathbb{R}$. A function $f: K \rightarrow \mathbb{R}$ is said to be $s$-preinvex with respect to $\eta$, if for all $u, v \in K, t \in[0,1]$ and $s \in(0,1]$, the following inequality holds

$$
f(u+t \eta(v, u)) \leq(1-t)^{s} f(u)+t^{s} f(v) .
$$

The function $f$ is said to be $s$-preincave if and only if $-f$ is $s$-preinvex.

1.8. Theorem. [42] Let $f: K=[a, a+\eta(b, a)] \subseteq[0, \infty) \rightarrow(0, \infty)$ be a s-preinvex function on the interval of the real numbers $K^{\circ}$ (the interior of $K$ ) and $a, b \in K^{\circ}$ with $\eta(b, a)>0$. Then the following inequalities holds:

$$
2^{s-1} f\left(\frac{2 a+\eta(b, a)}{2}\right) \leq \frac{1}{\eta(b, a)} \int_{a}^{a+\eta(b, a)} f(x) d x \leq \frac{f(a)+f(b)}{s+1} .
$$

For more recent results on Hermite-Hadamard type and Simpson's type inequalities for preinvex, log-preinvex functions, s-preinvex functions, prequasiinvex functions and $n$-times differentiable preinvex functions, we refer the interested readers to $[4,16,17,18$, 19, 35, 39, 40, 42].

The main purpose of the present paper is to establish new Hermite-Hadamard type inequalities in Section 2 that are connected with the right-side and left-side of HermiteHadamard inequality for $n$-times differentiable $s$-preinvex functions which generalize those results established for $n$-times differentiable preinvex functions and $n$-times differentiable convex functions.

\section{Main Results}

In order to prove our main results, we need the following lemmas:

2.1. Lemma. [16] Let $K \subseteq \mathbb{R}$ be an open invex subset with respect to $\eta: K \times K \rightarrow \mathbb{R}$. Suppose that $f: K \rightarrow \mathbb{R}$ is a function such that $f^{(n)}$ exists on $K$ for $n \in \mathbb{N}, n \geq 1$. If $f^{(n)}$ 
is integrable on $[a, a+\eta(b, a)]$, then for every $a, b \in K$ with $\eta(b, a)>0$, the following equality holds:

$$
\begin{gathered}
-\frac{f(a)+f(a+\eta(b, a))}{2}+\frac{1}{\eta(b, a)} \int_{a}^{a+\eta(b, a)} f(x) d x \\
+\sum_{k=2}^{n-1} \frac{(-1)^{k}(k-1)(\eta(b, a))^{k}}{2(k+1) !} f^{(k)}(a+\eta(b, a)) \\
=\frac{(-1)^{n-1}(\eta(b, a))^{n}}{2 n !} \int_{0}^{1} t^{n-1}(n-2 t) f^{(n)}(a+t \eta(b, a)) d t
\end{gathered}
$$

where the sum above takes 0 when $n=1$ and $n=2$.

2.2. Lemma. [16] Let $K \subseteq \mathbb{R}$ be an open invex subset with respect to $\eta: K \times K \rightarrow \mathbb{R}$. Suppose that $f: K \rightarrow \mathbb{R}$ is a function such that $f^{(n)}$ exists on $K$ for $n \in \mathbb{N}, n \geq 1$. If $f^{(n)}$ is integrable on $[a, a+\eta(b, a)]$, then for every $a, b \in K$ with $\eta(b, a)>0$, the following equality holds:

$$
\begin{array}{r}
\sum_{k=0}^{n-1} \frac{\left[(-1)^{k}+1\right](\eta(b, a))^{k}}{2^{k+1}(k+1) !} f^{(k)}\left(a+\frac{1}{2} \eta(b, a)\right)-\frac{1}{\eta(b, a)} \int_{a}^{a+\eta(b, a)} f(x) d x \\
=\frac{(-1)^{n+1}(\eta(b, a))^{n}}{n !} \int_{0}^{1} K_{n}(t) f^{(n)}(a+t \eta(b, a)) d t
\end{array}
$$

where

$$
K_{n}(t):=\left\{\begin{array}{ll}
t^{n}, & t \in\left[0, \frac{1}{2}\right] \\
(t-1)^{n}, & t \in\left(\frac{1}{2}, 1\right]
\end{array} .\right.
$$

We are now ready to present our first result.

2.3. Theorem. Let $K \subseteq[0, \infty)$ be an open invex subset with respect to $\eta: K \times K \rightarrow \mathbb{R}$. Suppose that $f: K \rightarrow \mathbb{R}$ is a function such that $f^{(n)}$ exists on $K$ and $f^{(n)}$ is integrable on $[a, a+\eta(b, a)]$ for $n \in \mathbb{N}, n \geq 2$. If $\left|f^{(n)}\right|^{q}$ is s-preinvex on $K$ for $q \geq 1$, then for every $a, b \in K$ with $\eta(b, a)>0$, we have the following inequality:

$$
\begin{aligned}
& \mid \frac{f(a)+f(a+\eta(b, a))}{2}-\frac{1}{\eta(b, a)} \int_{a}^{a+\eta(b, a)} f(x) d x \\
& -\sum_{k=2}^{n-1} \frac{(-1)^{k}(k-1)(\eta(b, a))^{k}}{2(k+1) !} f^{(k)}(a+\eta(b, a)) \mid \\
& \leq \frac{(\eta(b, a))^{n}}{2 n !}\left(\frac{n-1}{n+1}\right)^{1-\frac{1}{q}}\left(Q\left|f^{(n)}(a)\right|^{q}+P\left|f^{(n)}(b)\right|^{q}\right)^{\frac{1}{q}}
\end{aligned}
$$

where

$$
P=\frac{n(n-1)+s(n-2)}{(n+s)(n+s+1)}, Q=n B(n, s+1)-2 B(n+1, s+1)
$$

and

$$
B(x, y)=\int_{0}^{1} t^{x-1}(1-t)^{y-1}
$$

for $x>0, y>0$ is the Beta function. 
Proof. Suppose $n \geq 2$ and $q=1$. By $s$-preinvexity of $\left|f^{(n)}\right|$ on $K$ and lemma 2.1, we get

$$
\begin{aligned}
& \mid \frac{f(a)+f(a+\eta(b, a))}{2}-\frac{1}{\eta(b, a)} \int_{a}^{a+\eta(b, a)} f(x) d x \\
& -\sum_{k=2}^{n-1} \frac{(-1)^{k}(k-1)(\eta(b, a))^{k}}{2(k+1) !} f^{(k)}(a+\eta(b, a)) \mid \\
& \leq \frac{(\eta(b, a))^{n}}{2 n !} \int_{0}^{1} t^{n-1}(n-2 t)\left|f^{(n)}(a+t \eta(b, a))\right| d t \\
& \leq \frac{(\eta(b, a))^{n}}{2 n !} \int_{0}^{1} t^{n-1}(n-2 t)\left((1-t)^{s}\left|f^{(n)}(a)\right|+t^{s}\left|f^{(n)}(b)\right|\right) d t \\
& =\frac{(\eta(b, a))^{n}}{2 n !}\left(\left|f^{(n)}(b)\right| \int_{0}^{1} t^{n+s-1}(n-2 t) d t+\left|f^{(n)}(a)\right| \int_{0}^{1} t^{n-1}(n-2 t)(1-t)^{s} d t\right) .
\end{aligned}
$$

Since

$$
\int_{0}^{1} t^{n+s-1}(n-2 t) d t=\frac{n(n-1)+s(n-2)}{(n+s)(n+s+1)}=P
$$

and

$$
\begin{aligned}
\int_{0}^{1} t^{n-1}(n-2 t)(1-t)^{s} d t & =n \int_{0}^{1} t^{n-1}(1-t)^{s} d t-2 \int_{0}^{1} t^{n}(1-t)^{s} d t \\
& =n B(n, s+1)-2 B(n+1, s+1)=Q .
\end{aligned}
$$

Using the above observations in (2.4), we get (2.3). The proof for the case $q=1$ is complete.

Assume now that $q>1$, then by the $s$-preinvexity of $\left|f^{(n)}\right|^{q}$ on $K$, lemma 2.1 and the Hölder's inequality, we have

$$
\begin{aligned}
& \mid \frac{f(a)+f(a+\eta(b, a))}{2}-\frac{1}{\eta(b, a)} \int_{a}^{a+\eta(b, a)} f(x) d x \\
& -\sum_{k=2}^{n-1} \frac{(-1)^{k}(k-1)(\eta(b, a))^{k}}{2(k+1) !} f^{(k)}(a+\eta(b, a)) \mid \\
& \leq \frac{(\eta(b, a))^{n}}{2 n !}\left(\int_{0}^{1} t^{n-1}(n-2 t) d t\right)^{1-\frac{1}{q}}\left(\int_{0}^{1} t^{n-1}(n-2 t)\left|f^{(n)}(a+t \eta(b, a))\right|^{q} d t\right)^{\frac{1}{q}} \\
& \leq \frac{(\eta(b, a))^{n}}{2 n !}\left(\int_{0}^{1} t^{n-1}(n-2 t) d t\right)^{1-\frac{1}{q}} \\
& \quad \times\left(\left|f^{(n)}(b)\right|^{q} \int_{0}^{1} t^{n+s-1}(n-2 t) d t+\left|f^{(n)}(a)\right|^{q} \int_{0}^{1} t^{n-1}(n-2 t)(1-t)^{s}\right)^{\frac{1}{q}}
\end{aligned}
$$

which is the inequality (2.3). Hence the proof of the theorem is completed.

2.4. Remark. If in Theorem 2.3, we take $s=1$, we get Theorem 8 from [16]. 
2.5. Corollary. Suppose that the assumptions of Theorem 2.3 are satisfied. Then for $n=2$, we have

$$
\begin{aligned}
& \left|\frac{f(a)+f(a+\eta(b, a))}{2}-\frac{1}{\eta(b, a)} \int_{a}^{a+\eta(b, a)} f(x) d x\right| \\
& \quad \leq \frac{(\eta(b, a))^{2}}{2}\left(\frac{1}{6}\right)^{1-\frac{1}{q}}\left(\frac{\left|f^{\prime \prime}(a)\right|^{q}+\left|f^{\prime \prime}(b)\right|^{q}}{(s+3)(s+2)}\right)^{\frac{1}{q}} .
\end{aligned}
$$

2.6. Remark. If in Corollary $2.5 s=1$, we get a result proved in Corollary 1 from [16].

2.7. Remark. If in Theorem 2.3, we take $\eta(b, a)=b-a$. Then one gets a result proved in Theorem 1.1 from [11].

2.8. Theorem. Let $K \subseteq[0, \infty)$ be an open invex subset with respect to $\eta: K \times K \rightarrow \mathbb{R}$. Suppose that $f: K \rightarrow \mathbb{R}$ is a function such that $f^{(n)}$ exists on $K$ and $f^{(n)}$ is integrable on $[a, a+\eta(b, a)]$ for $n \in \mathbb{N}, n \geq 2$. If $\left|f^{(n)}\right|^{q}$ is s-preinvex on $K$ for $q \geq 1$, then for every $a, b \in K$ with $\eta(b, a)>0$, we have the following inequality:

$$
\begin{aligned}
& \mid \frac{f(a)+f(a+\eta(b, a))}{2}-\frac{1}{\eta(b, a)} \int_{a}^{a+\eta(b, a)} f(x) d x \\
& -\sum_{k=2}^{n-1} \frac{(-1)^{k}(k-1)(\eta(b, a))^{k}}{2(k+1) !} f^{(k)}(a+\eta(b, a)) \mid \\
& \leq \frac{(\eta(b, a))^{n}(n-1)^{1-1 / q}}{2 n !}\left[\{n B(n q-q+1, s+1)-2 B(n q-q+2, s+1)\}\left|f^{(n)}(a)\right|^{q}\right. \\
& \left.+\left\{\frac{n}{n q-q+s+1}-\frac{2}{n q-q+s+2}\right\}\left|f^{(n)}(b)\right|^{q}\right]^{1 / q},
\end{aligned}
$$

where

$$
B(x, y)=\int_{0}^{1} t^{x-1}(1-t)^{y-1}
$$

for $x>0, y>0$ is the Beta function.

Proof. The case when $q=1$ is easy to prove so we assume that $q>1$. By making use of Lemma 2.1, the Hölder inequality and the $s$-preinvexity of $\left|f^{(n)}\right|^{q}$, we have

$$
\begin{aligned}
& \mid \frac{f(a)+f(a+\eta(b, a))}{2}-\frac{1}{\eta(b, a)} \int_{a}^{a+\eta(b, a)} f(x) d x \\
& -\sum_{k=2}^{n-1} \frac{(-1)^{k}(k-1)(\eta(b, a))^{k}}{2(k+1) !} f^{(k)}(a+\eta(b, a)) \mid \leq \frac{(\eta(b, a))^{n}}{2 n !}\left(\int_{0}^{1}(n-2 t) d t\right)^{1-1 / q} \\
& \times\left[\int_{0}^{1} t^{q(n-1)}(n-2 t)\left((1-t)^{s}\left|f^{(n)}(a)\right|^{q}+t^{s}\left|f^{(n)}(b)\right|^{q}\right) d t\right]^{1 / q}=\frac{(\eta(b, a))^{n}(n-1)^{1-1 / q}}{2 n !} \\
& \times\left[\{n B(n q-q+1, s+1)-2 B(n q-q+2, s+1)\}\left|f^{(n)}(a)\right|^{q}\right. \\
& \left.\quad+\left\{\frac{n}{n q-q+s+1}-\frac{2}{n q-q+s+2}\right\}\left|f^{(n)}(b)\right|^{q}\right]^{1 / q} .
\end{aligned}
$$

This completes the proof of the theorem. 
2.9. Corollary. Suppose that the conditions of Theorem 2.8 are fulfilled and $n=2$. Then

$$
\begin{aligned}
& \mid \begin{array}{r}
\frac{f(a)+f(a+\eta(b, a))}{2}-\frac{1}{\eta(b, a)} \int_{a}^{a+\eta(b, a)} f(x) d x \mid \\
\leq \frac{(\eta(b, a))^{2}}{2^{2-1 / q}} \times\left[\{B(q+1, s+1)-B(q+2, s+1)\}\left|f^{\prime \prime}(a)\right|^{q}\right. \\
\left.\quad+\frac{\left|f^{\prime \prime}(b)\right|^{q}}{(q+s+1)(q+s+2)}\right]^{1 / q},
\end{array}
\end{aligned}
$$

where $B(x, y), x, y>0$ is the Beta's function.

2.10. Corollary. If we take $q=1$ and $s=1$ in Corollary 2.10. Then

$$
\begin{aligned}
\mid \frac{f(a)+f(a+\eta(b, a))}{2}-\frac{1}{\eta(b, a)} \int_{a}^{a+\eta(b, a)} & f(x) d x \mid \\
\leq & \frac{(\eta(b, a))^{2}}{24}\left[\left|f^{\prime \prime}(a)\right|^{q}+\left|f^{\prime \prime}(b)\right|\right] .
\end{aligned}
$$

2.11. Remark. For $\eta(b, a)=b-a$, we obtain new bounds of the difference between the middle and right-side of Hermite-Hadamard inequalities (1.1) in terms of second order derivatives.

Now we give some results related to left-side of Hermite-Hadamard's inequality for $n$-times differentiable $s$-preinvex functions.

2.12. Theorem. Let $K \subseteq[0, \infty)$ be an open invex subset with respect to $\eta: K \times K \rightarrow \mathbb{R}$. Suppose that $f: K \rightarrow \mathbb{R}$ is a function such that $f^{(n)}$ exists on $K$ and $f^{(n)}$ is integrable on $[a, a+\eta(b, a)]$. If $\left|f^{(n)}\right|^{q}$ is s-preinvex on $K$ for $q>1, n \in \mathbb{N}, n \geq 1, s \in(0,1]$, then for every $a, b \in K$ with $\eta(b, a)>0$, we have the following inequality:

$$
\begin{aligned}
& \mid \sum_{k=0}^{n-1} \frac{\left[(-1)^{k}+1\right](\eta(b, a))^{k}}{2^{k+1}(k+1) !} f^{(k)}\left(a+\frac{1}{2} \eta(b, a)\right) \\
& \quad-\frac{1}{\eta(b, a)} \int_{a}^{a+\eta(b, a)} f(x) d x \mid \leq \frac{(\eta(b, a))^{n}}{2^{n} n !(n p+1)^{\frac{1}{p}}}\left[\frac{\left|f^{(n)}(a)\right|^{q}+\left|f^{(n)}(b)\right|^{q}}{s+1}\right]^{\frac{1}{q}},
\end{aligned}
$$

where $\frac{1}{p}+\frac{1}{q}=1$.

Proof. Suppose $n \geq 1$. By using lemma 2.2 and the $s$-preinvexity of $\left|f^{(n)}\right|^{q}$ on $K$ for $n \in \mathbb{N}, n \geq 1, q>1$, we have

$$
\begin{gathered}
\left|\sum_{k=0}^{n-1} \frac{\left[(-1)^{k}+1\right](\eta(b, a))^{k}}{2^{k+1}(k+1) !} f^{(k)}\left(a+\frac{1}{2} \eta(b, a)\right)-\frac{1}{\eta(b, a)} \int_{a}^{a+\eta(b, a)} f(x) d x\right| \\
\leq \frac{(\eta(b, a))^{n}}{n !} \int_{0}^{1}\left|K_{n}(t)\right|\left|f^{(n)}(a+t \eta(b, a))\right| d t \\
\leq \frac{(\eta(b, a))^{n}}{n !}\left(\int_{0}^{1}\left|K_{n}(t)\right|^{p}\right)^{\frac{1}{p}}\left(\int_{0}^{1}\left|f^{(n)}(a+t \eta(b, a))\right|^{q} d t\right)^{\frac{1}{q}} .
\end{gathered}
$$


Since

$$
\int_{0}^{1}\left|K_{n}(t)\right|^{p}=\int_{0}^{\frac{1}{2}} t^{n p} d t+\int_{\frac{1}{2}}^{1}(1-t)^{n p} d t=\frac{1}{2^{n p}(n p+1)}
$$

and

$$
\begin{aligned}
\int_{0}^{1}\left|f^{(n)}(a+t \eta(b, a))\right|^{q} d t & \leq \int_{0}^{1}(1-t)^{s}\left|f^{(n)}(a)\right|^{q} d t+\int_{0}^{1} t^{s}\left|f^{(n)}(b)\right|^{q} d t \\
& =\frac{\left|f^{(n)}(a)\right|^{q}+\left|f^{(n)}(b)\right|^{q}}{s+1}
\end{aligned}
$$

An application of the above observations in (2.12), we get the desired inequality (2.11). This completes the proof of the theorem.

2.13. Corollary. Under the assumptions of Theorem 2.12, if $n=2$, then we obtain the following inequality:

$$
\begin{aligned}
\mid f\left(a+\frac{1}{2} \eta(b, a)\right)-\frac{1}{\eta(b, a)} \int_{a}^{a+\eta(b, a)} & f(x) d x \mid \\
& \leq \frac{(\eta(b, a))^{2}}{8(2 p+1)^{\frac{1}{p}}}\left[\frac{\left|f^{\prime \prime}(a)\right|^{q}+\left|f^{\prime \prime}(b)\right|^{q}}{s+1}\right]^{\frac{1}{q}},
\end{aligned}
$$

where $\frac{1}{p}+\frac{1}{q}=1$.

2.14. Corollary. In Corollary 2.13, if we take $s=1$, then one gets the following result:

$$
\begin{aligned}
\mid f\left(a+\frac{1}{2} \eta(b, a)\right)-\frac{1}{\eta(b, a)} \int_{a}^{a+\eta(b, a)} & f(x) d x \mid \\
& \leq \frac{(\eta(b, a))^{2}}{8(2 p+1)^{\frac{1}{p}}}\left[\frac{\left|f^{\prime \prime}(a)\right|^{q}+\left|f^{\prime \prime}(b)\right|^{q}}{2}\right]^{\frac{1}{q}},
\end{aligned}
$$

where $\frac{1}{p}+\frac{1}{q}=1$.

2.15. Corollary. In Theorem 2.12, if $\eta(b, a)=b-a$, we have the following inequality:

$$
\begin{aligned}
\mid \sum_{k=0}^{n-1} \frac{\left[(-1)^{k}+1\right](b-a)^{k}}{2^{k+1}(k+1) !} f^{(k)} & \left(\frac{a+b}{2}\right)-\frac{1}{b-a} \int_{a}^{b} f(x) d x \mid \\
& \leq \frac{(b-a)^{n}}{2^{n} n !(n p+1)^{\frac{1}{p}}}\left[\frac{\left|f^{(n)}(a)\right|^{q}+\left|f^{(n)}(b)\right|^{q}}{s+1}\right]^{\frac{1}{q}}
\end{aligned}
$$

where $\frac{1}{p}+\frac{1}{q}=1$.

A different approach leads us to the following result:

2.16. Theorem. Let $K \subseteq[0, \infty)$ be an open invex subset with respect to $\eta: K \times K \rightarrow \mathbb{R}$. Suppose that $f: K \rightarrow \mathbb{R}$ is a function such that $f^{(n)}$ exists on $K$ and $f^{(n)}$ is integrable 
on $[a, a+\eta(b, a)]$. If $\left|f^{(n)}\right|^{q}$ is s-preinvex on $K$ for $n \in \mathbb{N}, n \geq 1, q \in \mathbb{R}, q>1$ and $s \in(0,1]$, then for every $a, b \in K$ with $\eta(b, a)>0$, we have the following inequality:

$$
\begin{gathered}
\left|\sum_{k=0}^{n-1} \frac{\left[(-1)^{k}+1\right](\eta(b, a))^{k}}{2^{k+1}(k+1) !} f^{(k)}\left(a+\frac{1}{2} \eta(b, a)\right)-\frac{1}{\eta(b, a)} \int_{a}^{a+\eta(b, a)} f(x) d x\right| \\
\leq \frac{(\eta(b, a))^{n}}{2^{n+\frac{1}{p}}(n p+1)^{\frac{1}{p}} n !}\left[\left(\frac{\left(2^{s+1}-1\right)\left|f^{(n)}(a)\right|^{q}+\left|f^{(n)}(b)\right|^{q}}{2^{s+1}(s+1)}\right)^{\frac{1}{q}}\right. \\
\left.+\left(\frac{\left|f^{(n)}(a)\right|^{q}+\left(2^{s+1}-1\right)\left|f^{(n)}(b)\right|^{q}}{2^{s+1}(s+1)}\right)^{\frac{1}{q}}\right],
\end{gathered}
$$

where $\frac{1}{p}+\frac{1}{q}=1$.

Proof. From lemma 2.2 and the power-mean integral inequality, we have

$$
\begin{gathered}
\left|\sum_{k=0}^{n-1} \frac{\left[(-1)^{k}+1\right](\eta(b, a))^{k}}{2^{k+1}(k+1) !} f^{(k)}\left(a+\frac{1}{2} \eta(b, a)\right)-\frac{1}{\eta(b, a)} \int_{a}^{a+\eta(b, a)} f(x) d x\right| \\
\leq \frac{(\eta(b, a))^{n}}{n !}\left[\left(\int_{0}^{\frac{1}{2}} t^{n p} d t\right)^{\frac{1}{p}}\left(\int_{0}^{\frac{1}{2}}\left|f^{(n)}(a+t \eta(b, a))\right|^{q} d t\right)^{\frac{1}{q}}\right. \\
\left.+\left(\int_{\frac{1}{2}}^{1}(1-t)^{n p} d t\right)^{\frac{1}{p}}\left(\int_{\frac{1}{2}}^{1}\left|f^{(n)}(a+t \eta(b, a))\right|^{q} d t\right)^{\frac{1}{q}}\right] .
\end{gathered}
$$

Since $\left|f^{(n)}\right|^{q}$ is $s$-preinvex on $K$ in the second sense for $n \in \mathbb{N}, n \geq 1, q \in \mathbb{R}, q>1$ and $s \in(0,1]$. Hence for every $a, b \in K$ with $\eta(b, a)>0$, we have

$$
\begin{aligned}
\int_{0}^{\frac{1}{2}} t^{n}\left|f^{(n)}(a+t \eta(b, a))\right|^{q} d t & \leq\left|f^{(n)}(a)\right|^{q} \int_{0}^{\frac{1}{2}}(1-t)^{s} d t+\left|f^{(n)}(b)\right|^{q} \int_{0}^{\frac{1}{2}} t^{s} d t \\
& =\frac{2^{s+1}-1}{2^{s+1}(s+1)}\left|f^{(n)}(a)\right|^{q}+\frac{1}{2^{s+1}(s+1)}\left|f^{(n)}(b)\right|^{q}
\end{aligned}
$$

and

$$
\begin{aligned}
\int_{0}^{\frac{1}{2}}\left|f^{(n)}(a+t \eta(b, a))\right|^{q} d t & \leq\left|f^{(n)}(a)\right|^{q} \int_{\frac{1}{2}}^{1}(1-t)^{s} d t+\left|f^{(n)}(b)\right|^{q} \int_{\frac{1}{2}}^{1} t^{s} d t \\
& =\frac{1}{2^{s+1}(s+1)}\left|f^{(n)}(a)\right|^{q}+\frac{2^{s+1}-1}{2^{s+1}(s+1)}\left|f^{(n)}(b)\right|^{q}
\end{aligned}
$$

Also

$$
\int_{0}^{\frac{1}{2}} t^{n p} d t=\int_{\frac{1}{2}}^{1}(1-t)^{n p} d t=\frac{1}{2^{n p+1}(n p+1)}
$$

Using (2.18), (2.19) and (2.20) in (2.17), we get the required inequality (2.16). This completes the proof of the theorem.

2.17. Remark. For $s=1$, Theorem 2.16 becomes Theorem 11 from [16]. 
2.18. Corollary. For $s=1$ and $n=2$, we get the following inequality from [16]:

$$
\begin{aligned}
& f\left(a+\frac{1}{2} \eta(b, a)\right)-\frac{1}{\eta(b, a)} \int_{a}^{a+\eta(b, a)} f(x) d x \mid \\
\leq \frac{(\eta(b, a))^{2}}{8 \cdot 2^{\frac{1}{p}}(2 p+1)^{\frac{1}{p}}}\left[\left(\frac{3\left|f^{\prime \prime}(a)\right|^{q}+\left|f^{\prime \prime}(b)\right|^{q}}{8}\right)^{\frac{1}{q}}\right. & \left.+\left(\frac{\left|f^{\prime \prime}(a)\right|^{q}+3\left|f^{\prime \prime}(b)\right|^{q}}{8}\right)^{\frac{1}{q}}\right]
\end{aligned}
$$

where $\frac{1}{p}+\frac{1}{q}=1$.

2.19. Theorem. Let $K \subseteq[0, \infty)$ be an open invex subset with respect to $\eta: K \times K \rightarrow \mathbb{R}$. Suppose that $f: K \rightarrow \mathbb{R}$ is a function such that $f^{(n)}$ exists on $K$ and $f^{(n)}$ is integrable on $[a, a+\eta(b, a)]$. If $\left|f^{(n)}\right|^{q}$ is s-preinvex on $K$ for $n \in \mathbb{N}, n \geq 1, q \in \mathbb{R}, q \geq 1$ and $s \in(0,1]$, then for every $a, b \in K$ with $\eta(b, a)>0$, we have the following inequality:

$$
\begin{gathered}
\left|\sum_{k=0}^{n-1} \frac{\left[(-1)^{k}+1\right](\eta(b, a))^{k}}{2^{k+1}(k+1) !} f^{(k)}\left(a+\frac{1}{2} \eta(b, a)\right)-\frac{1}{\eta(b, a)} \int_{a}^{a+\eta(b, a)} f(x) d x\right| \\
\leq \frac{(\eta(b, a))^{n}(n+1)^{\frac{1}{q}}}{2^{(n+1)\left(1-\frac{1}{q}\right)}(n+1) !}\left[\left(L\left|f^{(n)}(a)\right|^{q}+M\left|f^{(n)}(b)\right|^{q}\right)^{\frac{1}{q}}\right. \\
\left.+\left(M\left|f^{(n)}(a)\right|^{q}+N\left|f^{(n)}(b)\right|^{q}\right)^{\frac{1}{q}}\right]
\end{gathered}
$$

where

$$
\begin{aligned}
& L=B\left(\frac{1}{2} ; n+1, s+1\right), M=\frac{1}{2^{n+s+1}(n+s+1)}, \\
& N=B(s+1, n+1)-B\left(\frac{1}{2} ; s+1, n+1\right) \\
& B(x, y)=\int_{0}^{1} t^{x-1}(1-t)^{y-1}
\end{aligned}
$$

for $x>0, y>0$ is the Beta function and

$$
B(z ; x, y)=\int_{0}^{z} t^{x-1}(1-t)^{y-1}
$$

is the generalized of the Beta function $B(x, y)$. 
Proof. It is not difficult to see that (2.22) holds true for $q=1$. Suppose that $q>1$. From lemma 2.2 and the Hölder's integral inequality, we have

$$
\begin{aligned}
& \left|\sum_{k=0}^{n-1} \frac{\left[(-1)^{k}+1\right](\eta(b, a))^{k}}{2^{k+1}(k+1) !} f^{(k)}\left(a+\frac{1}{2} \eta(b, a)\right)-\frac{1}{\eta(b, a)} \int_{a}^{a+\eta(b, a)} f(x) d x\right| \\
& \leq \frac{(\eta(b, a))^{n}}{n !}\left[\left(\int_{0}^{\frac{1}{2}} t^{n} d t\right)^{1-\frac{1}{q}}\left(\int_{0}^{\frac{1}{2}} t^{n}\left|f^{(n)}(a+t \eta(b, a))\right|^{q} d t\right)^{\frac{1}{q}}\right. \\
& \left.+\left(\int_{\frac{1}{2}}^{1}(1-t)^{n} d t\right)^{1-\frac{1}{q}}\left(\int_{\frac{1}{2}}^{1}(1-t)^{n}\left|f^{(n)}(a+t \eta(b, a))\right|^{q} d t\right)^{\frac{1}{q}}\right] .
\end{aligned}
$$

Since $\left|f^{(n)}\right|^{q}$ is $s$-preinvex on $K$ in the second sense for $n \in \mathbb{N}, n \geq 1, q \in \mathbb{R}, q \geq 1$ and $s \in(0,1]$. Hence for every $a, b \in K$ with $\eta(b, a)>0$, we have

$$
\begin{gathered}
\int_{0}^{\frac{1}{2}} t^{n}\left|f^{(n)}(a+t \eta(b, a))\right|^{q} d t \leq\left|f^{(n)}(a)\right|^{q} \int_{0}^{\frac{1}{2}} t^{n}(1-t)^{s} d t+\left|f^{(n)}(b)\right|^{q} \int_{0}^{\frac{1}{2}} t^{n+s} d t \\
=B\left(\frac{1}{2} ; n+1, s+1\right)\left|f^{(n)}(a)\right|^{q}+\frac{\left|f^{(n)}(b)\right|^{q}}{2^{n+s+1}(n+s+1)}
\end{gathered}
$$

and

$$
\begin{aligned}
& \int_{0}^{\frac{1}{2}}(1-t)^{n}\left|f^{(n)}(a+t \eta(b, a))\right|^{q} d t \\
& \leq\left|f^{(n)}(a)\right|^{q} \int_{\frac{1}{2}}^{1}(1-t)^{n+s} d t+\left|f^{(n)}(b)\right|^{q} \int_{\frac{1}{2}}^{1} t^{s}(1-t)^{n} d t \\
& =\frac{\left|f^{(n)}(a)\right|^{q}}{2^{n+s+1}(n+s+1)}+\left[B(s+1, n+1)-B\left(\frac{1}{2} ; s+1, n+1\right)\right]\left|f^{(n)}(b)\right|^{q} .
\end{aligned}
$$

Using (2.24), (2.25) and

$$
\int_{0}^{\frac{1}{2}} t^{n} d t=\int_{\frac{1}{2}}^{1}(1-t)^{n} d t=\frac{1}{2^{n+1}(n+1)}
$$

we get the required inequality (2.22). This completes the proof of the theorem.

2.20. Corollary. If we choose $n=2$ and $s=1$ in the Theorem 2.19, we get the following inequality:

$$
\begin{aligned}
& f\left(a+\frac{1}{2} \eta(b, a)\right)-\frac{1}{\eta(b, a)} \int_{a}^{a+\eta(b, a)} f(x) d x \mid \\
\leq \frac{(\eta(b, a))^{2} \cdot 3^{\frac{1}{q}-1}}{2^{4-\frac{3}{q}}}\left[\left(\frac{5\left|f^{\prime \prime}(a)\right|^{q}+3\left|f^{\prime \prime}(b)\right|^{q}}{192}\right)^{\frac{1}{q}}\right. & \\
& \left.+\left(\frac{3\left|f^{\prime \prime}(a)\right|^{q}+5\left|f^{\prime \prime}(b)\right|^{q}}{192}\right)^{\frac{1}{q}}\right] .
\end{aligned}
$$


Proof. Since for $n=2$ and $s=1, L=B\left(\frac{1}{2} ; 3,2\right)=\frac{5}{192}, M=\frac{1}{64}$ and $N=B(2,3)-$ $B\left(\frac{1}{2} ; 2,3\right)=\frac{5}{192}$ and hence proof follows.

\section{Applications to Special Means}

In the following we give certain generalizations of some notions for a positive valued function of a positive variable.

3.1. Definition. [3]A function $M: \mathbb{R}_{+}^{2} \rightarrow \mathbb{R}_{+}$, is called a Mean function if it has the following properties:

(1) Homogeneity: $M(a x, a y)=a M(x, y)$, for all $a>0$,

(2) Symmetry: $M(x, y)=M(y, x)$,

(3) Reflexivity: $M(x, x)=x$,

(4) Monotonicity: If $x \leq x^{\prime}$ and $y \leq y^{\prime}$, then $M(x, y) \leq M\left(x^{\prime}, y^{\prime}\right)$,

(5) Internality: $\min \{x, \bar{y}\} \leq M(x, y) \leq \max \{x, y\}$.

We consider some means for arbitrary positive real numbers $\alpha, \beta$ (see for instance [3]).

(1) The arithmetic mean:

$$
A:=A(\alpha, \beta)=\frac{\alpha+\beta}{2}
$$

(2) The The geometric mean:

$$
G:=G(\alpha, \beta)=\sqrt{\alpha \beta}
$$

(3) The harmonic mean:

$$
H:=H(\alpha, \beta)=\frac{2}{\frac{1}{\alpha}+\frac{1}{\beta}}
$$

(4) The power mean:

$$
P_{r}:=P_{r}(\alpha, \beta)=\left(\frac{\alpha^{r}+\beta^{r}}{2}\right)^{\frac{1}{r}}, r \geq 1
$$

(5) The identric mean:

$$
I:=I(\alpha, \beta)= \begin{cases}\frac{1}{e}\left(\frac{\beta^{\beta}}{\alpha^{\alpha}}\right), & \alpha \neq \beta \\ \alpha, & \alpha=\beta\end{cases}
$$

(6) The logarithmic mean:

$$
L:=L(\alpha, \beta)=\frac{\alpha-\beta}{\ln |\alpha|-\ln |\beta|}, \quad|\alpha| \neq|\beta|
$$

(7) The generalized log-mean:

$$
L_{p}:=L_{p}(\alpha, \beta)=\left[\frac{\beta^{p+1}-\alpha^{p+1}}{(p+1)(\beta-\alpha)}\right], \alpha \neq \beta, p \in \mathbb{R} \backslash\{-1,0\} .
$$

It is well known that $L_{p}$ is monotonic nondecreasing over $p \in \mathbb{R}$, with $L_{-1}:=L$ and $L_{0}:=I$. In particular, we have the following inequality $H \leq G \leq L \leq I \leq A$.

Now, let $a$ and $b$ be positive real numbers such that $a<b$. Consider the function $M:=M(a, b):[a, a+\eta(b, a)] \times[a, a+\eta(b, a)] \rightarrow \mathbb{R}^{+}$, which is one of the above mentioned means, therefore one can obtain various inequalities for these means as follows: 
Setting $\eta(b, a)=M(b, a)$ in (2.6), (2.13), one can obtain the following interesting inequalities involving means:

$$
\begin{aligned}
& \left|\frac{f(a)+f(a+M(b, a))}{2}-\frac{1}{M(b, a)} \int_{a}^{a+M(b, a)} f(x) d x\right| \\
& \leq \frac{(M(b, a))^{2}}{2}\left(\frac{1}{6}\right)^{1-\frac{1}{q}}\left(\frac{\left|f^{\prime \prime}(a)\right|^{q}+\left|f^{\prime \prime}(b)\right|^{q}}{(s+3)(s+2)}\right)^{\frac{1}{q}} . \\
& \left|f\left(a+\frac{1}{2} M(b, a)\right)-\frac{1}{M(b, a)} \int_{a}^{a+M(b, a)} f(x) d x\right| \\
& \mid \leq \frac{(M(b, a))^{2}}{8(2 p+1)^{\frac{1}{p}}}\left[\frac{\left|f^{\prime \prime}(a)\right|^{q}+\left|f^{\prime \prime}(b)\right|^{q}}{s+1}\right]^{\frac{1}{q}},
\end{aligned}
$$

Letting $M=A, G, H, P_{r}, I, L, L_{p}$ in (3.1) and in (3.2), we get the inequalities involving means for a particular choice of a twice differentiable $s$-preinvex function $f$, and the details are left to the interested reader.

\section{References}

[1] Antczak, T. Mean value in invexity analysis, Nonl. Anal., 60 (2005), 1473-1484.

[2] Avci, M. Kavurmaci, H. and Özdemir, M.E. New inequalities of Hermite-Hadamard type via s-convex functions in the second sense with applications, Applied Mathematics and Computation, 217 (2011) 5171-5176.

[3] Bullen, P.S. Handbook of Means and Their Inequalities, Kluwer Academic Publishers, Dordrecht, 2003.

[4] Barani, A., Ghazanfari, A.G. and Dragomir S.S. Hermite-Hadamard inequality through prequsiinvex functions, RGMIA Research Report Collection, 14(2011), Article 48, 7 pp.

[5] Barani, A., Ghazanfari, A.G. and Dragomir S.S. Hermite-Hadamard inequality for functions whose derivatives absolute values are preinvex, RGMIA Research Report Collection, 14(2011), Article 64, 11 pp.

[6] Ben-Israel, A. and Mond, B. What is invexity?, J. Austral. Math. Soc., Ser. B, 28(1986), No. 1, 1-9.

[7] Dragomir, S.S. and Agarwal, R.P. Two inequalities for differentiable mappings and applications to special means of real numbers and trapezoidal formula, Appl. Math. Lett., 11(5) (1998), 91-95.

[8] Dragomir, S.S. and Pearce, C.E.M. Selected Topics on Hermite-Hadamard Inequalities and Applications, RGMIA Monographs, Victoria University, 2000.

[9] Dragomir, S.S. and Fitzpatrick, S. The Hadamard's inequality for s-convex functions in the second sense, Demonstratio Math., 32 (4) (1999), 687-696.

[10] Hudzik, H. and Maligranda, L. Some remraks on s-convex functions, Aequationes Math. 48 (1994), 100-111.

[11] Jiang, W.-D., Niu, D.-W, Hua, Y. and Qi, F. Generalizations of Hermite-Hadamard inequality to $n$-time differentiable functions which are s-convex in the second sense, Analysis (Munich) 32 (2012), 1001-1012; Available online at http://dx.doi.org/10.1524/anly.2012.1161.

[12] Hanson, M.A. On sufficiency of the Kuhn-Tucker conditions, J. Math. Anal. Appl. 80 (1981) 545-550.

[13] Wang, S.-H., Xi, B.-Y and Qi, F. Some new inequalities of Hermite-Hadamard type for n-times differentiable functions which are m-convex, Analysis (Munich) 32 (2012), no. 3, 247-262; Available online at http://dx.doi.org/10.1524/anly.2012.1167.

[14] Hadamard, J. Étude sur les propriétés des fonctions entières et en particulier d'une fonction considerée par Riemann, J. Math Pures Appl., 58 (1893), 171-215. 
[15] Hwang, D.-Y. Some inequalities for n-time differentiable mappings and applications, Kyugpook Math. J. 43(2003), 335-343.

[16] Latif, M.A. On Hermite-Hadamard type integral inequalities for n-times differentiable preinvex functions with applications, Stud. Univ. Babeş-Bolyai Math. 58(2013), No. 3, 325-343.

[17] Latif, M.A. and Dragomir, S.S. Some weighted integral inequalities for differentiable preinvex and prequasiinvex functions with applications, Journal of Inequalities and Applications 2013, 2013:575.

[18] Latif, M.A. Some inequalities for prequasiinvex functions with applications, Konurlap Journal of Math.Vol. 1, no 2, 17-29.

[19] Iscan, I. Ostrowski type inequalites for functions whose derivatives are preinvex, arXiv:1204.2010v1.

[20] Kırmac1, U.S. Inequalities for differentiable mappings and applications to special means of real numbers and to midpoint formula, Appl. Math. Comp., 147 (2004), 137-146.

[21] Kırmacı, U.S. and Özdemir, M.E. On some inequalities for differentiable mappings and applications to special means of real numbers and to midpoint formula, Appl. Math. Comp., 153 (2004), 361-368.

[22] Kirmac1, U.S. Improvement and further generalization of inequalities for differentiable mappings and applications, Computers and Math. with Appl., 55 (2008), 485-493.

[23] Kırmacı, U.S., Bakula, M.K., Özdemir, M.E. and Pečarić, J. Hadamard-type inequalities for s-convex functions, Appl. Math. and Comput., Volume 193, Issue 1( 2007), Pages 26-35.

[24] Mohan, S.R. and Neogy, S.K. On invex sets and preinvex functions, J. Math. Anal. Appl. 189 (1995), 901-908.

[25] Noor, M.A. Hermite-Hadamard integral inequalities for log-preinvex functions, J. Math. Anal. Approx. Theory, 2(2007), 126-131.

[26] Noor, M.A. Variational like inequalities, Optimization, 30(1994), 323-330.

[27] Noor, M.A. On Hadamard integral inequalities involving two log-preinvex functions, J. Inequal. Pure Appl. Math., 8(2007), No. 3, 1-6, Article 75.

[28] Noor, M.A. Hadamard integral inequalities for product of two preinvex function, Nonl. Anal. Forum, 14 (2009), 167-173.

[29] Pearce, C.E.M. and Pečarić, J. Inequalities for differentiable mappings with application to special means and quadrature formulae, Appl. Math. Lett., 13(2) (2000), 51-55.

[30] Pini, R. Invexity and generalized Convexity, Optimization 22 (1991) 513-525.

[31] Sarikaya, M.Z., Saglam, A. and Yıldırım, H. New inequalities of Hermite-Hadamard type for functions whose second derivatives absolute values are convex and quasi-convex, International Journal of Open Problems in Computer Science and Mathematics ( IJOPCM), 5(3), 2012.

[32] Sarikaya, M.Z., Saglam, A. and Yıldırım, H. On some Hadamard-type inequalities for $h$ convex functions, Journal of Mathematical Inequalities, Volume 2, Number 3 (2008), 335341.

[33] Sarikaya, M.Z., Avci, M. and Kavurmaci, H. On some inequalities of Hermite-Hadamard type for convex functions, ICMS Iternational Conference on Mathematical Science. AIP Conference Proceedings 1309, 852 (2010).

[34] Sarikaya, M.Z. and Aktan, N. On the generalization some integral inequalities and their applications, Mathematical and Computer Modelling, Volume 54, Issues 9-10, November 2011, Pages 2175-2182.

[35] Sarikaya, M.Z., Bozkurt, H. and Alp, N. On Haermite-Hadamard type integral inequalities for preinvex and log-preinvex functions, Contemporary Analysis and Applied Mathematics Vol.1, No.2, 237-252, 2013.

[36] Sarikaya, M.Z., Set, E. and Özdemir, M.E. On new inequalities of Simpson's type for sconvex functions, Computers and Mathematics with Applications, 60(2010), 2191-2199.

[37] Saglam, A., Sarikaya, M.Z. Yildirim H. Some new inequalities of Hermite-Hadamard's type, Kyungpook Mathematical Journal, 50(2010), 399-410.

[38] Weir, T. and Mond, B. Preinvex functions in multiobjective optimization, Journal of Mathematical Analysis and Applications, 136 (1998) 29-38. 
[39] Wang, Y., Wang, S. -H. and Qi, F. Simpson type integral inequalities in which the power of the absolute value of the first derivative of the integrand is s-preinvex, Facta Univ. (NIŠ), Ser. Math. Inform. Vol. 28, No 2 (2013), 151-159.

[40] Wang, Y., Xi, B.-Y. and Qi, F. Hermite-Hadamard type integral inequalities when the power of the absolute value of the first derivative of the integrand is preinvex, Le Matematiche. (to appear)

[41] Yang, X. M. and Li, D. On properties of preinvex functions, J. Math. Anal. Appl. 256 (2001) 229-241.

[42] Jue-You, L. On Hadamard-type inequalities for s-preinvex functions, Journal of Chongqing Normal University (Natural Science) 27(2010). 
